\title{
Magneto-Optical Study of Ferromagnetic Nanostructured Materials and Thin Films
}

\author{
R. V. Pisarev, V. V. Pavlov, and A. V. Kimel \\ A. F. Ioffe Physical Technical Institute of the Russian Academy of Sciences, 194021 St. Petersburg, Russia
}

\begin{abstract}
Experimental observations of linear and nonlinear magneto-optical phenomena in magnetically ordered nanostructured materials and thin films are discussed. Films of ferromagnetic nanoparticles of CoNbTa in $\mathrm{SiO}_{2}$ matrix with a various relation of metal and dielectric phases were studied by means of static and dynamic magneto-optical techniques with subpicosecond temporal resolution. The optical second harmonic generation was investigated in ferromagnetic-semiconductor heterostructures $\mathrm{MnAs} / \mathrm{Si}(111)$. The observed changes in the second-harmonic intensity were odd in magnetisation. Second and third harmonic optical spectra were studied in epitaxial magnetic thin films in the spectral range of $1.7-3.2 \mathrm{eV}$ and $2.4-4.2 \mathrm{eV}$, respectively. No significant increase of the intensity of the nonlinear spectra was found above the bandgap near $3.2 \mathrm{eV}$ where linear absorption increases by two orders of magnitude.
\end{abstract}

Key words: nanostructured materials, magnetooptical effects, second harmonic generation, photoinduced phenomena, thin magnetic films

\section{Introduction}

Nanostructured magnetic materials and thin ferromagnetic films grown on semiconducting and insulating substrates are promising candidates for developing new memory systems and magneto-electronic devices. Among potential applications of these structures it is important to mention the possibility of developing devices combining the energy-independent magnetic memory or magnetosensor cells and semiconducting readout elements in the same integrated circuit, as well as other devices based on galvano-magnetic phenomena. The technical parameters of these devices will depend not only on the bulk properties of a ferromagnetic film, but also on the magnetic state of the film near the surface, and in particular near the ferromagnet-semiconductor interface.

Obviously further progress in developing new devices strongly depends on new materials and structures and methods of their characterisation because in many cases conventional tools for measuring magnetic properties become inefficient or difficult to apply. The magnetooptical Kerr effect (MOKE) was proven to be an efficient tool for studying magnetic properties of surfaces and thin films. In recent years, the magnetization-induceded second harmonic generation (MSHG) was proposed for studying the surface and interface magnetic states of thin-film structures [1]. These two magneto-optical methods, linear and nonlinear, mutually complement each other in a sense that linear magneto-optical Kerr effect is formed by a region having thickness on the order of the light penetration depth, whereas magnetic SHG is sensitive to the magnetic state of several atomic monolayers at the surface or in the vicinity of the interface.

In this paper we review results on linear and nonlinear magneto-optical study of thin films formed by ferromagnetic nanoparticles CoNbTa in the $\mathrm{SiO}_{2}$ matrices, ferromagnetic-semiconductor nanostructures of the $\mathrm{MnAs} / \mathrm{Si}$ type and epitaxial magnetic garnet films.

\section{CoNbTa-SiO 2 nanoparticle films}

In the last years composite nanoparticle materials on the basis of $\mathrm{Fe}, \mathrm{Co}, \mathrm{Ni}$ and other transition metal ions have attracted a great interest. First of all, it is caused by the giant magneto-resistance (GMR) phenomenon observed in these materials [2]. Besides, nanocomposite materials are characterised by a series of other unusual magnetic, electrical, optical and magneto-optical properties. Nonlinear optical studies of nanocomposite materials have shown a strong enhancement of optical nonlinearities [3], a correlation between magnetotransport and nonlinear optical properties [4].

\subsection{Growth and structure of samples}

Films of ferromagnetic granules of an amorphous alloy on the basis of cobalt, niobium and tantalum in a dielectric matrix of silicon oxide with the various contents of the metal phase, representing typical percolation systems, were deposited on ceramic substrates by the ion-beam sputtering method [5]. The size of metal granules was in the range of 2-5 nm. Metal nanoparticles formed a granular, not connected electrically structure up to a threshold and conducting cluster structure above the threshold. Three composites on a basis silicon oxide with CoNbTa particles and the amorphous film of a metal alloy not containing the oxide were investigated (see Table 1).

\subsection{Experimental results and discussion}

The study of static magnetic properties of nanoparticle films was based on a longitudinal MOKE technique described in [6]. A femtosecond Ti:sapphire laser operating in the photon energy range $1.45-1.7 \mathrm{eV}$ and a continuous helium-neon laser $(\hbar \omega=1.96 \mathrm{eV})$ have been used as light sources. The MOKE measurements were performed at the $45^{\circ}$ of incidence of the light beam. The magnetic field to $15 \mathrm{kOe}$ could be applied to the sample. 
Table 1. Parameters of nanoparticle films CoNbTa in $\mathrm{SiO}_{2}$ matrix.

\begin{tabular}{|c|c|c|c|}
\hline Sample & Composition & $\mathrm{SiO}_{2}$ contents & Thickness $(\mu \mathrm{m})$ \\
\hline$A$ & $\mathrm{Co}_{86.1} \mathrm{Nb}_{12.2} \mathrm{Ta}_{1.7}$ & without $\mathrm{SiO}_{2}$ & 5.0 \\
\hline$B$ & $\mathrm{Co}_{53.4} \mathrm{Nb}_{8.3} \mathrm{Ta}_{1.1}$ & $\mathrm{Si}_{20.2} \mathrm{O}_{17}$ & 4.6 \\
\hline$C$ & $\mathrm{Co}_{47.3} \mathrm{Nb}_{7.0} \mathrm{Ta}_{1.4}$ & $\mathrm{Si}_{23.6} \mathrm{O}_{20.7}$ & 4.7 \\
\hline$D$ & $\mathrm{Co}_{27.7} \mathrm{Nb}_{3.0} \mathrm{Ta}_{0.7}$ & $\mathrm{Si}_{26.7} \mathrm{O}_{41.9}$ & 4.6 \\
\hline
\end{tabular}

Theoretical [7, 8], and later experimental [9] works have shown, that the magnetisation of a medium can be achieved not only by an external magnetic field, but also by the electric field component of the light wave. However, until recently that possibility was used for studying diamagnetic and paramagnetic materials [10] and only recently for studying magnetically ordered materials [11]. In such experiments the all-optical technique of studying the ultrafast magnetic phenomena is used, where more intense beam is used as an optical pump, whereas a less intense beam is used as a probe. The probe beam monitors the state of a medium after the optical excitation by the pump beam. Such technique enables one to study the dynamic phenomena with the time resolution limited by the pulse duration. The magneto-optical Kerr rotation induced in the pump-probe experiment can be described as follows:

$$
\theta_{K}=-\frac{1}{n\left(n^{2}-1\right) \int_{-\infty}^{+\infty}\left|E_{0}\right|^{2} d t} \int_{-\infty}^{+\infty}\left(p^{(+)}-p^{(-)}\right) E_{0}^{*} d t
$$

where $n$ is the refractive index of a medium, $E_{0}$ is an electric field of the pump pulse; $p^{(1)}$ and $p^{()}$are thirdorder nonlinear polarisations, induced by the rightcircular-polarised pump pulse and the right (left)circular-polarised probe pulse. We call this phenomenon as a photo-induced magneto-optical Kerr effect [12].

Fig. 1 shows field dependencies of the MOKE for films $\mathrm{C}$ and $\mathrm{D}$. They are characterised by a narrow hysteresis and a saturation in magnetic fields of $15 \mathrm{kOe}$. Small values of saturation fields indicate that nanoparticles CoNbTa imbedded in the matrix $\mathrm{SiO}_{2}$ are

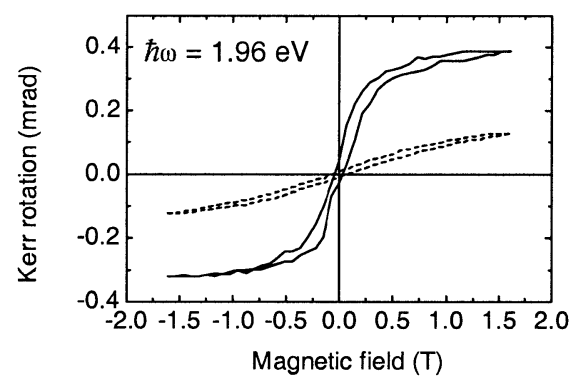

Fig. 1 Field dependences of the static longitudinal Kerr effect in films (solid line) and D (dashed line). ferromagnetic. No noticeable dispersion was revealed in the spectral range under study (Fig. 2). Nonmonotonic behaviour of the MOKE as a function of the Co content with a maximum at the cobalt concentration of $\sim 45 \%$ has been observed. Note, that in granular films the value of MOKE is by a factor of two-three larger, than in a metal alloy.

Measurements of the photo-induced magneto-optical Kerr effect in the nanoparticle CoNbTa films have shown, that the signal is observed only in the time domain where the pump and probe pulses are overlapping. In nanogranule structures the considerable contribution to the nonlinear optical effects and in particular to the dynamic magneto-optical Kerr effect, may arise from the enhancement of the local field during overlapping of the pulses. After the termination of the pump pulse effects of the local field quickly damp due to the loss of optical coherence. This fact may be the reason that the dynamic Kerr effect is observed only during overlapping the pump and probe pulses. Another factor which can explain such behaviour may be a short lifetime of nonequilibrium spin polarisation. The reason for that can be a small lifetime of electrons in the excited states [13], and/or a small lifetime of spin excitations [14].

Spectual dependencies of the dynamic magnetooptical Kerr effect are shown in Fig. 3. Contrary to the static effect, the dynamic effect shows strong variations in all nanogranule structures. Spectral dependencies specify the presence of an electronic transitions in the region of $1.45 \mathrm{eV}$ or at lower energies outside the spectral range of the Ti:sapphire laser. It is necessary to

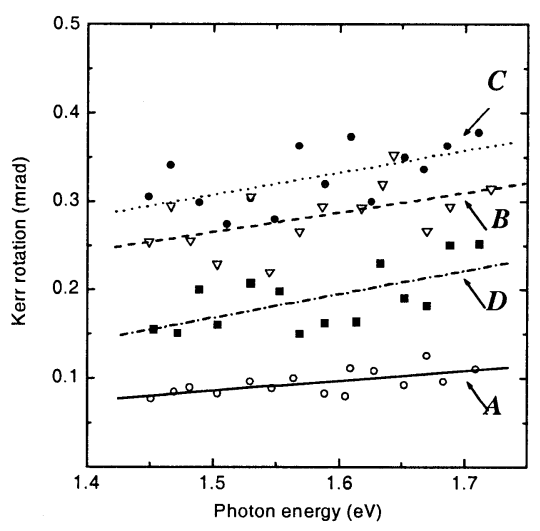

Fig. 2 Spectral dependences of the static longitudinal Kerr effect for films A, B, C and $\mathrm{D}$ in magnetic field of $3 \mathrm{kOe}$. Lines show results of linear approximation of experimental data indicated by points. 


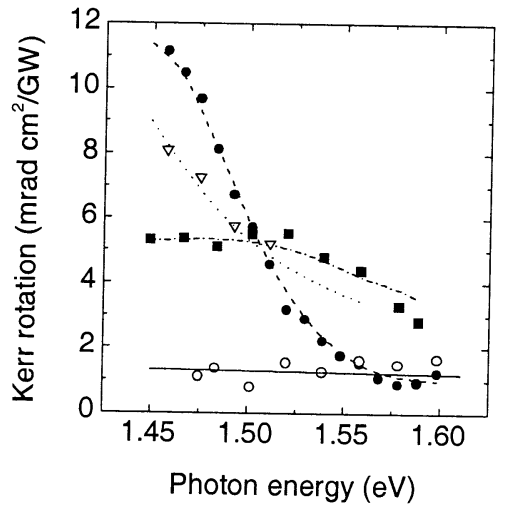

Fig. 3 Spectral dependences of the dynamic Kerr effect for films A (empty circles), B (empty triangles), $\mathrm{C}$ (black circles) and D (black squares). Lines show results of linear approximation of experimental data.

note, that in monolithic CoNbTa film the dynamic Kerr effect does not practically depend on the photon energy. It is obvious, that the electronic transition close to 1.45 $\mathrm{eV}$ or at lower energy is caused by the processes occurring in nanogranules CoNbTa. This can be established only by a method based on measurements of dynamic magneto-optical Kerr effect. In our opinion the resonant behaviour of the nonlinear magneto-optical signal close to $1.45 \mathrm{eV}$ is caused by effects of the local field enhancement caused by the plasmon resonance in the CoNbTa nanogranules.

\section{MnAs/Si (111) heterostructures}

Heterostructures obtained by growing ferromagnetic films directly on the surface of semiconductors such as silicon or gallium arsenide are promising for developing new magnetoelectronic devices [15]. We report on the study of MOKE and SHG in ferromagnet-semiconductor heterostructures $\mathrm{CaF}_{2} / \mathrm{MnAs} / \mathrm{Si}(111)$ prepared by a MBE method $[6,16]$. The MnAs film thickness was in the range of 40-70 nm [6], or 6-12 $\mathrm{nm}$ [16]. A morphology of films was studied with a use of an atomic force microscope and was found to be strongly sensitive to the film preparation conditions. The X-ray results for the MnAs/Si(111) film (MnAs film thickness $d=9 \mathrm{~nm}$ ) are shown in Fig. 4. One can see intense $\mathrm{Si}(111)$ and $\mathrm{Si}(222)$ peaks in addition to $\mathrm{MnAs}[0001]$ (at $15.5^{\circ}$ ), $\mathrm{MnAs}[0002]$

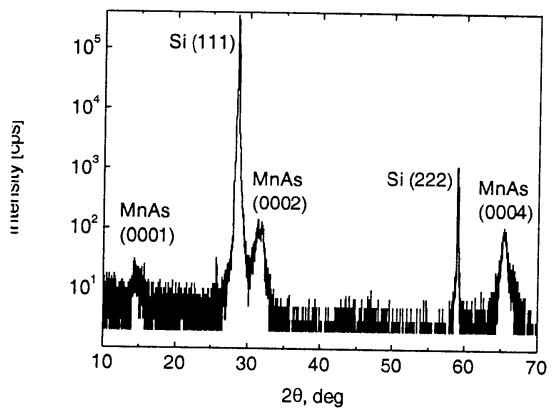

Fig. 4 X-ray diffraction spectrum of a MnAs/Si((111) film (d=9 nm). (at $31.3^{\circ}$ ) and $\mathrm{MnAs}$ [0004] (at 65.3 ${ }^{\circ}$ ) peaks. These peaks indicate that the film grows along the c-axis. The average thickness (d) was defined as the amount of material deposited on the Si substrate.

\subsection{SHG in MnAs/Si (111) heterostructures}

Using the nonlinear optical setup described in [6], we have measured SHG in MnAs/Si(111) heterostructures. Fig. 5 shows rotational anisotropy measurements of SHG in configurations, where maximum magnetisationinduced SHG contributions have been observed. All measurements have been done at a single wavelength $(\lambda$ $=800 \mathrm{~nm}$ ).

In the electric-dipole approximation, the nonlinear polarisation $\mathbf{P}^{2 \omega}$ induced by the electric field $\mathbf{E}^{\omega}$ of an incident electromagnetic wave in a medium with the magnetisation $\mathbf{M}$ can be written as a sum of two terms:

$$
\mathbf{P}^{2 \omega}=\varepsilon_{0}\left(\chi^{(2)}+\hat{\chi}^{(3)} \mathbf{M}\right) \mathbf{E}^{\omega} \mathbf{E}^{\omega},
$$

where $\chi^{(2)}$ and $\chi^{(3)}$ are nonlinear tensors describing the crystallographic and magnetic contributions to the nonlinear polarisation, respectively. $\hat{\chi}^{(2)}$ is a third-rank polar tensor, and $\hat{\chi}^{(3)}$ is an axial tensor of rank four. Both tensors differ from zero only in noncentrosymmetric media. Consequently, the generation of the second optical harmonic in the bulk of $\mathrm{CaF}_{2}, \mathrm{MnAs}$, and $\mathrm{Si}$ possessing a centrosymmetric structure is forbidden in the clcctric-dipole approximation. The nonlınear polarisation can differ from zero only in the vicinity of interfaces such as air/ $\mathrm{CaF}_{2}, \mathrm{CaF}_{2} / \mathrm{MnAs}$, and $\mathrm{MnAs} / \mathrm{Si}$, where the space inversion is broken. Nonvanishing components of the tensors $\hat{\chi}^{(2)}$ and $\hat{\chi}^{(3)}$ for the $\mathrm{CaF}_{2} / \mathrm{MnAs}$ and $\mathrm{MnAs} / \mathrm{Si}(111)$ interfaces is given in [6]. Rotational anisotropy patterns shown in Fig. 5 reflect the symmetries of these interfaces.

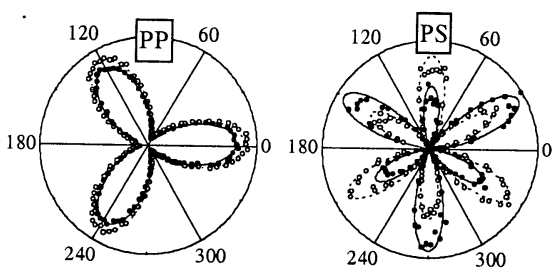

(a)

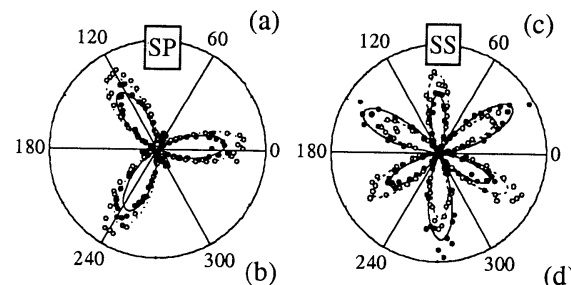

(d)

Fig. 5 Azimuthal dependencies of the SHG intensity in the $\mathrm{CaF}_{2} / \mathrm{MnAs}(40 \mathrm{~nm}) / \mathrm{As} / \mathrm{Si}(111)$ heterostructure for the $(a, b)$ equatorial and $(c, d)$ meridional geometries and for different combinations of the input-output polarisations of radiation: (a) PP, (b) PS, (c) SP, and (d) SS. Dark circles correspond to $I^{2 \omega}(+\mathrm{M})$, and open circles to $I^{2 \omega}(-\mathrm{M})$.

Trans. Magn. Soc. Jpn., Vol. 2, No. 4, 2002 


\section{YIG, Bi-YIG magnetic garnet films}

Bulk magnetic garnets and epitaxial magnetic garnet films are two well known groups of materials characterised by a large variety of very useful magnetic, acoustic, optical, and magneto-optical properties [17]. Bulk crystals of magnetic garnets belong to the centrosymmetric cubic point group $m 3 m$ (space group $I a 3 d)$. However, in thin films the observation of a linear magneto-electric effect proved, that the inversion symmetry is broken [18]. Thus, SHG is allowed in the electric-dipole approximation in thin garnet films. Previous studies of SHG in magnetic garnet films were restricted to a few selected photon energies determined by the pump lasers, such as $1.17 \mathrm{eV}$ (Nd:YAG lasers) or around $1.5 \mathrm{eV}$ (Ti-sapphire lasers) [19]. In order to disclose the microscopical mechanisms of nonlinear optical susceptibilities in magnetic garnets the spectroscopic studies of second and third harmonic generation (TGH) were undertaken. We studied films of yttrium iron garnet YIG and bismuth-substituted YIG (Bi-YIG) grown on gadolinium gallium garnet $\mathrm{Gd}_{3} \mathrm{Ga}_{5} \mathrm{O}_{12}$ (GGG) or substituted GGG (SGGG) substrates with different crystallographic orientations (111), (110), and (210).

Optical absorption spectra were measured using a Cary 2300 spectrophotometer and were found to be in agreement with literature data. Absorption spectra measured at $\mathrm{T}=15 \mathrm{~K}$ in three films are shown in Fig. 6. Optical densities higher than $\mathrm{D}=4.5$ are above the working range of the spectrophotometer and could not be measured.

\subsection{SHG spectra in magnetic garnet films}

SHG setup based on a Nd:YAG laser system and a $\beta$ $\mathrm{BaB}_{2} \mathrm{O}_{4}$ operated optical parametric oscillator was described in [20]. Second harmonic spectra were studied in the spectral range of 1.7-3.2 eV. Results on SHG in three different films are shown in Fig. 7 for two opposite orientations of the magnetisation $\mathbf{M}$ in the transverse geometry. A split transition near $2.4 \mathrm{eV}$ is well resolved for the YIG/GGG(111) film (see Fig. 7(a)). Magnetic contrast, determined as a normalised difference between SHG intensities for the two opposite directions of magnetisation, varies from 0 to $100 \%$. Some features in the $\mathrm{SH}$ spectrum can be assigned to the crystal-field

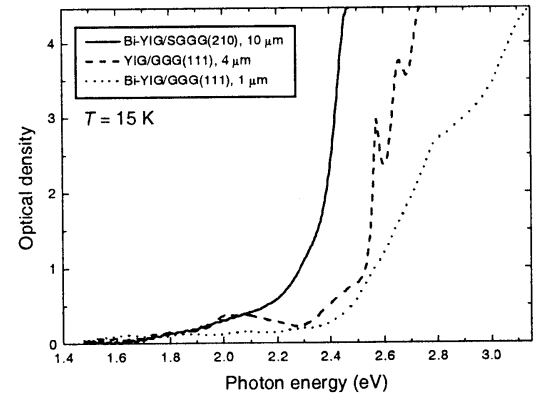

Fig. 6 Optical absorption spectra in the three garnet films transitions in the two iron sublattices [21]. Two other sharp absorption features near $2.57 \mathrm{eV}$ (presumably due to the ${ }^{6} \mathrm{~A}_{1} \rightarrow{ }^{4} \mathrm{E},{ }^{4} \mathrm{~A}_{1}$ transition in the tetrahedral sublattice) and $2.66 \mathrm{eV}$ (presumably due to the ${ }^{6} \mathrm{~A}_{1 \mathrm{~g}} \rightarrow{ }^{4} \mathrm{E}_{\mathrm{g}},{ }^{4} \mathrm{~A}_{1 \mathrm{~g}}$ transition in the octahedral sublattice) are also observed in the SHG spectrum (see Fig. 7(a)). Two more spectral features in the absorption spectrum are observed at 2.9 and $3.2 \mathrm{eV}$, with the oscillator strength by an order of magnitude higher than those for the tetrahedral transitions. However, the relevant features in the SH spectrum are of the same order of magnitude as for the transitions with the lower optical absorption.

As a rule bismuth-substituted films show the strongest SH signals [19]. Two types of bismuthsubstituted samples have been studied. SHG spectra for Bi-YIG/GGG(111) and Bi-YIG/SGGG(210) are shown in Fig. 7(b, c). Three (see Fig. 7(b)) and five (see Fig. 7 (c)) strong bands of varying magnetic contrast could be well resolved. Similar to the YIG/GGG(111) film the increase of the linear optical absorption does not lead to a noticeable increase of the $\mathrm{SH}$ intensity. That could be due to the fact, that only localized $d-d$ transitions contribute to nonlinear optical spectra.

\subsection{THG spectra in magnetic garnet films}

Third harmonic optical spectra were studied in the spectral range of 2.4-4.2 eV. Results on THG in three different films are shown in Fig. 8. Note that even in the electric-dipole approximation a breaking of space inversion symmetry is not required for the observation of a THG signal. Although the optical absorption and the magnitudes of the magneto-optical signals are very different in the three films, their third harmonic spectra

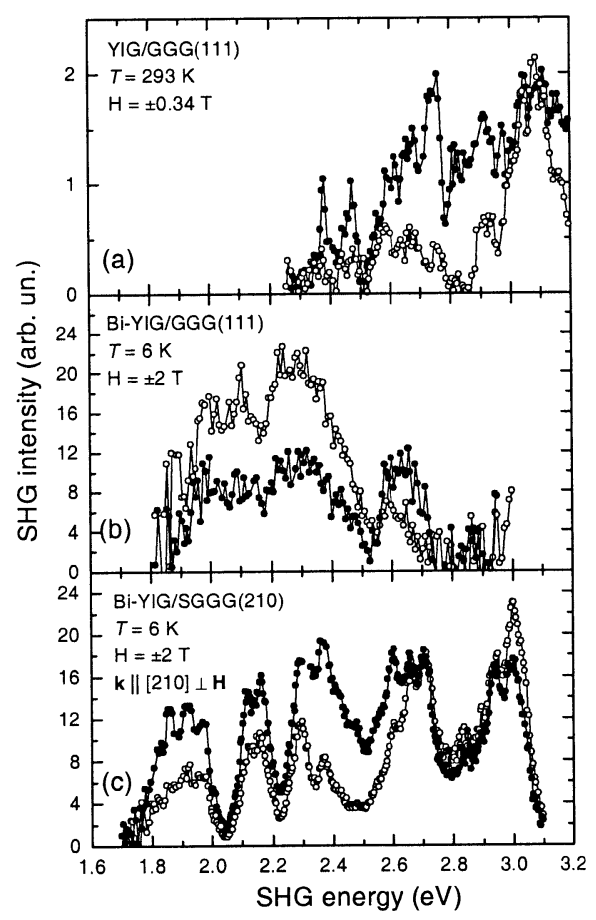

Fig. 7 SHG in the three garnet films 
are similar. The tetrahedral transition ${ }^{6} \mathrm{~A}_{1} \rightarrow{ }^{4} \mathrm{~T}_{2}$ centered near $2.4 \mathrm{eV}$ is well resolved in the THG spectrum, and in particular in the YIG/GGG(111) film. Note that the strong increase of the linear absorption above $3 \mathrm{eV}$ is not accompanied by a similar increase of the THG spectra. Contrary to the SHG spectra, no difference in the THG spectra is observed within the experimental accuracy upon a reorientation of the magnetization or upon a variation of the magnetic field. This is surprising, because according to a phenomenological analysis magnetic contributions to the THG are allowed both in longitudinal and transverse geometries.

\section{Conclusions}

Thin films of ferromagnetic nanocomposite CoNbTa in a $\mathrm{SiO}_{2}$ matrix with various contents of cobalt were studied by static and dynamic magneto-optical methods. Spectral and concentration dependencies of static and photo-induced magneto-optical Kerr effects were obtained.

SHG from MnAs/Si heterostructures was studied. The sources of nonlinear signals were determined from the experimental azimuthal dependencies of the SHG intensity. The changes of the SHG signals in magnetic field were found to be odd in magnetization.

SHG spectra of various anisotropic magnetic garnet films were measured in a range of photon energies stretching from 1.7-3.2 eV and thus, below the fundamental bandgap at about $3.2 \mathrm{eV}$. The spectra revealed contributions of nonmagnetic and magnetic type to the total SHG intensity. We also report the THG spectra in the range of 2.4-4.2 eV and thus, below and above the bandgap. While the intensity of linear absorption grows progressively as a function of photon energy, the intensity of the SHG and THG spectra does not show a similar behaviour.

\section{Acknowledgements}

We are grateful to D. Fröhlich, M. Fiebig, K. Kohn, Th. Rasing and M. Tanaka for fruitful collaboration that resulted in this work. This work was supported by the Russian Foundation for Basic Research, and the Ministry of Sciences.

\section{References}

1) H. A. Wierenga, W. de Jong, M. W. J. Prins, Th. Rasing, R. Vollmer, A. Kirilyuk, H. Schwabe, and J. Kirschner: Phys. Rev. Lett. 74, 1462 (1995)

2) A. E. Berkovitz, J. R. Mitchell, M. J. Carey, A. P. Young, S. Zhang, F. E. Spada, F. T. Parker, A. Hutten, and G. Thomas: Phys. Rev. Lett., 68, 3745 (1992).

3) V. M. Shalaev and A. K. Sarychev: Phys. Rev. B, 57, 13265 (1998).

4) T. V. Murzina, T. V. Misuryaev, A. F. Kravets, J. Güdde, D. Schuhmacher, G. Marowsky, A. A. Nikulin, and O. A. Aktsipetrov: Surface Science, 482-485, 1101 (2001).

5) A. V. Kimel, R. V. Pisarev, A. A. Rzhevsky,
Yu. E. Kalinin, A.V.Sitnikov, O.V.Stognej, F. Bentivegna, and Th. Rasing: Phys. Solid State, 44 (2002) (to be published)

6) A. G. Banshchikov, A. V. Kimel', V. V. Pavlov, R. V. Pisarev, N. S. Sokolov, and Th. Rasing: Physics of the Solid State, 42, 909 (2000).

7) L. P. Pitaevskii: Sov. Phys. JETP, 39, 1450 (1960).

8) L. D. Landau and E. M. Lifshitz, Electrodynamics of Continuous Media (Pergamon Press, Oxford, 1984).

9) P. van der Ziel, P. S. Pershan, L. D. Malmstrom: Phys. Rev. Lett., 15, 190 (1965).

10) Optical Orientation, Modern Problems in Condensed Matter Science, edited by F. Meier and B. P. Zacharchenya (North-Holland, Amsterdam, 1984), Vol. 8.

11) Ganping Ju, A. Vertikov, A. V. Nurmikko, C. Canady and Gang Xiao, R. F. C. Farrow and A. Cebollada: Phys. Rev. $B$ 57, R700 (1998).

12) A. V. Kimel, V. V. Pavlov, R. V. Pisarev, V. N. Gridnev, F. Bentivegna and Th. Rasing: Phys. Rev. B, 62, R10610 (2000), Phys. Rev. B, 63, 235201 (2001).

13) V. Kimel, R. V. Pisarev, F. Bentivegna, and Th. Rasing: Phys. Rev. B, 64, 201103(R) (2001).

14) L. V. Lutsev: Phys. Solid State, 44, 102 (2002).

15) G. A. Prinz: Science, 250, 1092 (1990); Physics Today, 48 (4), 58 (1995).

16) A. G. Banshchikov, A. V. Kimel, R. V. Pisarev, A. A. Rzhevsky, N. S. Sokolov, A. Keen, Th. Rasing, A. M. Nazmul, and M. Tanlaka: Phys. Solid State, 43, 1864 (2001).

17) Landolt-Börnstein, Numerical Data and Functional Relationships in Science and Technology, New Series, Group III, 27/e (Springer-Verlag, Berlin, 1991).

18) B. B. Krichevtsov, V. V. Pavlov, and R. V. Pisarev: JETP Lett. 49, 535 (1989); Sov. Phys. Solid State, 31, 1142 (1989).

19) V. N. Gridnev, V. V. Pavlov, R. V. Pisarev, A. Kirilyuk, and Th. Rasing: Phys. Rev. B, 63, 184407 (2001) and references therein.

20) C. Degenhardt, M. Fiebig, D. Fröhlich, Th. Lottermoser, and R. V. Pisarev: Appl. Phys. B, 73, 139 (2001).

21) V. V. Pavlov, R. V. Pisarev, M. Fiebig, and D. Fröhlich: Low Temp. Phys., 28 (2002) (to be published).

Received June 15, 2002; Accepted July 19, 2002. 